\title{
GPRC5A: An emerging prognostic biomarker for predicting malignancy of Pancreatic Cancer based on bioinformatics analysis
}

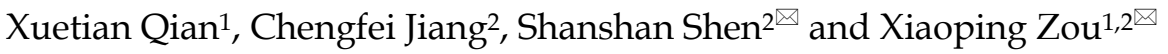 \\ 1. Nanjing Drum Tower Hospital Clinical College of Nanjing Medical University, Nanjing, 210008, People's Republic of China. \\ 2. Department of Gastroenterology, The Affiliated Drum Tower Hospital of Nanjing University Medical School, Nanjing, 210008, People's Republic of China. \\ $\triangle$ Corresponding authors: Xiaoping Zou, E-mail: zouxp@nju.edu.cn; Shanshan Shen, E-mail: shenshanshan@njglyy.com.
}

(c) The author(s). This is an open access article distributed under the terms of the Creative Commons Attribution License (https://creativecommons.org/licenses/by/4.0/). See http://ivyspring.com/terms for full terms and conditions.

Received: 2020.08.29; Accepted: 2021.01.06; Published: 2021.02.02

\begin{abstract}
Background: Pancreatic cancer $(\mathrm{PaCa})$ is a highly lethal malignancy. The treatment options for $\mathrm{PaCa}$ lack efficacy. The study aimed to explore the molecular biomarkers for predicting survival of $\mathrm{PaCa}$ and identify the potential carcinogenic mechanisms of the selected gene.

Methods: Based on public databases of $\mathrm{PaCa}$, differentially expressed genes (DEGs) were identified using Networkanalyst. Survival analyses were exerted on GEPIA. Oncomine and The Human Protein Atlas were used for verifying the expression on mRNA and protein levels. Enrichment analyses were generated on Metascape and gene set enrichment analysis (GSEA). Univariate analyses were performed to determine the clinical factors associated with the expression of GPRC5A.

Results: GPRC5A was identified as the most valuable gene in predicting survival of $\mathrm{PaCa}$ patients. Patients with high expression of GPRC5A showed larger tumor size, higher TNM stages, higher tumor grade, and more positive resection margin. In mutant KRAS, TP53, CDKN2A and SMAD4 group, the expression of GPRC5A was higher than non-mutant group. Mechanistically, GPRC5A may promote metastasis of $\mathrm{PaCa}$ mainly via regulating epithelial-mesenchymal transition (EMT) and neuroactive ligand-receptor interaction.
\end{abstract}

Conclusion: GPRC5A may act as an oncogene in the progression of $\mathrm{PaCa}$ and could be a prognostic biomarker in predicting survival of $\mathrm{PaCa}$.

Key words: GPRC5A, pancreatic cancer, metastasis, epithelial-mesenchymal transition, neuroactive ligand-receptor interaction

\section{Introduction}

Pancreatic cancer (PaCa) is a highly lethal malignancy, with a 5-year survival rate less than $9 \%$ [1]. It has been predicted to rank the second cause of cancer-associated mortality by 2030 [2]. Surgical resection is the dominated treatment for $\mathrm{PaCa}$, yet the recurrence was observed in $72 \%$ of patients after operation [3]. Early metastasis is a key characteristic of $\mathrm{PaCa}$, which in part leads to tumor recurrence and makes most patients lose surgical opportunities [4]. In pathological issue, $\mathrm{PaCa}$ is characterized by apparent tumor desmoplasia, reduction in intra-tumoral vessel density and neural remodelling [5]. Thus, PaCa shows resistance to radiotherapy, chemotherapy, and molecular targeted therapy. The emerging immunotherapy also demonstrates little efficacy against $\mathrm{PaCa}$ [6]. On the other hand, $\mathrm{PaCa}$ is a molecularly diverse disease, driven by aberrations in at least 10 core signaling pathways [7]. Currently, molecular subtyping seems to be a new option for redefining the biology of $\mathrm{PaCa}$ and advancing therapeutic development [8].

To date, multiple microarray and sequencing datasets of $\mathrm{PaCa}$ are able to access in public. These datasets can be applied to screen genetic changes in tumorigenesis and identify potential genes with prognostic values. Meanwhile, the abundant 
information of the datasets has not been explored in detail, especially "The Cancer Genome Atlas" (TCGA), which contains millions of data of mutations, RNA sequencing (RNA-Seq), clinical information, copy number alteration and methylation. Bioinformatic mining methods are evolving rapidly in recent years, which make it possible for globally observing and deeply understanding the tumor biology in molecular level. Indeed, applying bioinformatic methods to deeply analyze datasets of $\mathrm{PaCa}$ would be beneficial for identifying the key genes underlined occurrence and progression of PaCa.

In the present study, we analyzed the differentially expressed genes (DEGs) patterns between $\mathrm{PaCa}$ tissue and normal pancreas, together with conducted survival analyses of the DEGs. Consequently, GPRC5A was identified as the most valuable gene in predicting survival of $\mathrm{PaCa}$ patients. Patients with high expression of GPRC5A showed larger tumor size, higher Tumor Node Metastasis (TNM) stages, higher tumor grade, and more positive resection margin. Mechanistically, GPRC5A may act as an oncogene in the progression of $\mathrm{PaCa}$ mainly via regulating epithelial-mesenchymal transition (EMT) and neuroactive ligand-receptor interaction. In summary, we preliminarily elucidated that GPRC5A could promote proliferation and metastasis of $\mathrm{PaCa}$, and further validated work is needed.

\section{Materials and Methods}

\section{Data collection and DEGs extraction}

The selection criteria for datasets related to $\mathrm{PaCa}$ were as follows: 1 . gene expression analysis was performed using the microarray technology or RNA-Seq technique; 2 . human samples were tested. The datasets, i.e., GSE15471, GSE16515 and GSE101448, were downloaded from Gene Expression Omnibus (GEO) (http://www.ncbi.nlm.nih.gov/ geo/) [9-16]. NetworkAnalyst (http://www. networkanalyst.ca) was used to identify DEGs of GSE15471, GSE16515 and GSE101448 and GEPIA databases (http://gepia.cancer-pku.cn/index.html) were utilized for extracting DEGs from The Cancer Genome Atlas (TCGA) plus Genotype Tissue Expression (GTEx) datasets between $\mathrm{PaCa}$ and normal samples [17-22]. The criteria for DEGs were adjusted $P$-value (adj. $P$ ) $<0.01$ and $\mid \log$ Fold Change $\quad(|\log \quad F C|)>2.0$. A comprehensive assessment of common DEGs was then performed.

\section{Kaplan-Meier survival analysis}

The overall survival (OS) and disease-free survival (DFS) curves of the previously identified common DEGs in PaCa were generated by GEPIA under the quartile group cut-off. Statistically significant difference was considered when a log-rank $P$ value $<0.05$ [17]. The genes were selected for further analysis when both of the log-rank $P$ values of OS and DFS were $<0.05$.

\section{GPRC5A expression in mRNA and protein level}

Five datasets (Ishikawa Pancreas, IacobuzioDonahue Pancreas 2, Segara Pancreas, Grutzmann Pancreas and Buchholz Pancreas) derived from Oncomine database (https://www.oncomine.org) were used to verify GPRC5A mRNA expression [23-28]. The protein expression of GPRC5A in PaCa and normal samples was verified by the immunohistochemical (IHC) result from The Human Protein Atlas (https:/ / www.proteinatlas.org) [29].

\section{GPRC5A mRNA expression under different clinicopathological variables and mutant genes}

The relative expression of GPRC5A across tumor and normal samples, as well as in various tumor sub-groups based on individual cancer stages, tumor grade, gender, race, age, chronic pancreatitis status, KRAS mutant status, TP53 mutant status, CDKN2A mutant status and SMAD4 mutant status was analyzed based on TCGA plus GTEx datasets [30].

\section{Functional enrichment analyses of DEGs between GPRC5A high-expression and low-expression group}

The raw expression data was downloaded from TCGA database including $179 \mathrm{PaCa}$ samples. The samples from metastatic tissues or of which the histological diagnosis discrepancy with $\mathrm{PaCa}$ were excluded. $177 \mathrm{PaCa}$ samples were included for further analysis. Depending on the expression of GPRC5A, patients were divided into 2 groups: the top $25 \%$ (high expression, $n=45$ ) and the last $25 \%$ of patients (low expression, $\quad \mathrm{n}=45$ ). $\quad$ NetworkAnalyst (http://www.networkanalyst.ca) was utilized to screen DEGs between GPRC5A high-expression and low-expression groups. Genes with adj. $P<0.05$ and $|\log \mathrm{FC}|)>1.0$ were considered as DEGs.

Biological interpretation of the screened DEGs was finished by computational functional analyses on Metascape (http://metascape.org) [31], which is an online analytical tool based on several bioinformatics resources (Gene Ontology (GO), Kyoto Encyclopedia of Genes and Genomes (KEGG) and Reactome).

\section{Gene set enrichment analysis (GSEA)}

GSEA software was exerted between GPRC5A high-expression and low-expression groups to analyze the enrichment results [32]. The gene sets with nominal $P<0.05$, | normalized enrichment score | 
$(|\mathrm{NES}|)>1.0$ and false discovery rate $(\mathrm{FDR})<25 \%$ were considered as statistically significant.

\section{Association between clinical characteristics and GPRC5A}

The clinical characteristic data were derived from TCGA database. Univariate analyses were performed to determine the clinical factors associated with mRNA expression of GPRC5A.

\section{Statistical analysis}

IBM SPSS V.23 Software (Statistical Package for the Social Sciences, IBM, New York, USA) was used for the statistical analysis. $X^{2}$ or Fisher's exact tests were used to compare distribution of categorical factors between the different groups. An unpaired Student $\mathrm{t}$ test or a Mann-Whitney test was used for comparisons of two groups. $P<0.05$ was considered statistically significant.

\section{Results}

\section{High expression of GPRC5A was associated with a poor DFS and OS in $\mathrm{PaCa}$}

As the procedure shown in Fig. 1A, we extracted DEGs from GSE15471, GSE16515, GSE101448 and TCGA plus GTEx datasets and exerted survival analyses on the common DEGs. Based on the survival results, we picked the gene with the most significant prognostic value for further analysis. 213, 285 and 309 genes respectively from GSE15471, GSE16515, and GSE101448 datasets were identified as DEGs based on $|\log \mathrm{FC}|>2.0$ and $P<0.01 .2608$ DEGs were extracted from TCGA plus GTEx datasets based on the same criteria above via another online tool GEPIA. 46 DEGs were marked as common changed genes between the four datasets, including 31 up-regulated DEGs (TRIM29, MMP11, GPX2, IFI27, SERPINB5, C19ORF33, KRT17, TFF1, SFN, AHNAK2, ITGA2, CTSE, LAMC2, CEACAM5, IGFBP3, LCN2, GPRC5A, SLC6A14, GABRP, ITGB6, POSTN, CST1, COL11A1, COMP, S100P, GJB2, KRT19, SULF1, CTHRC1, COL10A1 and COL8A1) and 15 down-regulated DEGs (ALB, CTRL, SERPINI2, AQP8, TMED6, NMT, CELA2B, ANPEP, RBPJL, ERP27, SPX, KLK1, EGF, TMEM52 and CBS) (Fig. 1B-C). The values of log FCcancer vs. normal, were shown in Fig. 1C.

The survival information of the 46 common DEGs were analyzed on GEPIA. Of them, 12 genes were meaningful in predicting overall survival and disease free survival, namely GPRC5A, C19ORF33, SERPINB5, ITGB6, TRIM29, ITGA2, LAMC2, S100P, KRT19, AHNAK2, KRT17 and GABRP (Fig. 1D). Among them, GPRC5A and SERPINB5 had the most significant value in DFS and OS, respectively (GPRC5A in DFS: $P=9.20 \mathrm{E}-05$; SERPINB5 in OS:
$P=3.70 \mathrm{E}-05)$. In addition, ITGB6 and KRT19 also showed great values in predicting poor OS of $\mathrm{PaCa}$ (ITGB6 in OS: $P=1.70 \mathrm{E}-05$; KRT19 in OS: $P=2.00 \mathrm{E}-04$ ). Of note, high expression of GPRC5A was both correlated with a poor DFS (log-rank $P=9.20 \mathrm{E}-05$, $\mathrm{HR}=4.6$ ) and $\mathrm{OS}$ (log-rank $P=0.0026, \mathrm{HR}=2.5$ ) (Fig. 1E). $G$ protein-coupled receptor class $C$ group 5 member A (GPRC5A), also known as retinoic acid-induced gene 3 (RAI3) or retinoic acid-induced gene 1 (RAIG1) is a member of class $C$ orphan $G$ protein-coupled receptors (GPCRs) [33]. Recently, studies have reported that it could promote malignancy of $\mathrm{PaCa}$ [34-36].

\section{Higher mRNA and protein expression of GPRC5A in PaCa compared with normal pancreas tissues}

Next, we validated the expression level of GPRC5A in tumors and normal tissues of $\mathrm{PaCa}$ in more datasets based on Oncomine and The Human Protein Atlas. As shown in Fig. 2A-E, in Ishikawa, Segara, Iacobuzio-Donahue, Grutzmann and Buchholz studies, GPRC5A was ranked in top 1\%, 2\%, $4 \%, 4 \%$ and $11 \%$ over-expression genes with the fold change of 2.505, 8.452, 18.047, 10.324 and 1.671, respectively. Besides, the protein expression of GPRC5A in tumor was higher than in normal pancreas validated via IHC on the online tool The Human Protein Atlas. Typical images of the IHC results were shown in Fig. 2F.

\section{GPRC5A transcription in subgroups of patients with $\mathrm{PaCa}$}

We analyzed the expression of GPRC5A in different subgroups of PaCa to see whether GPRC5A was correlated with some typical factor. Compared with the normal group, both female and male patients had significantly higher expression of GPRC5A $(P<0.001)$. Between the female and male patient group, no statistical difference was shown (Fig. 3A). In Fig. 3B, patients in all races, i.e., Caucasian, Asian and African American, showed higher GPRC5A expression than normal $(P<0.001)$. Same as above, there was no statistical difference between the races. In terms of age, except the group of 21-40 yrs, patients in the other three groups showed higher expression of GPRC5A than normal $(P<0.001)$. Between the PaCa subgroups of age, no difference was shown (Fig. 3C). Based on TNM stages, we found that patients in each stage had higher expression than normal pancreas $(P<0.001)$. Besides, the expression of GPRC5A in Stage II was significantly higher than Stage I $(P<0.001)$. An increasing trend of GPRC5A expression was observed from Stage I to Stage IV (Fig. 3D). As shown in Fig. 3E, in the tumor grade subgroups, the GPRC5A 
transcription of grade1-3 was all higher than the normal group. Both in $\mathrm{PaCa}$ patients with pancreatitis or without pancreatitis, GPRC5A expression was higher than normal pancreas. Of note, between the two groups, patients with pancreatitis showed higher GPRC5A expression than those without pancreatitis $(P<0.05)$ (Fig. 3F).
A

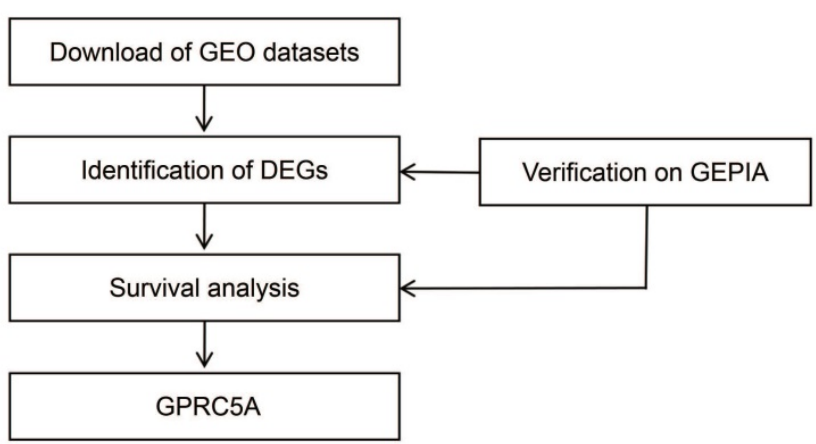

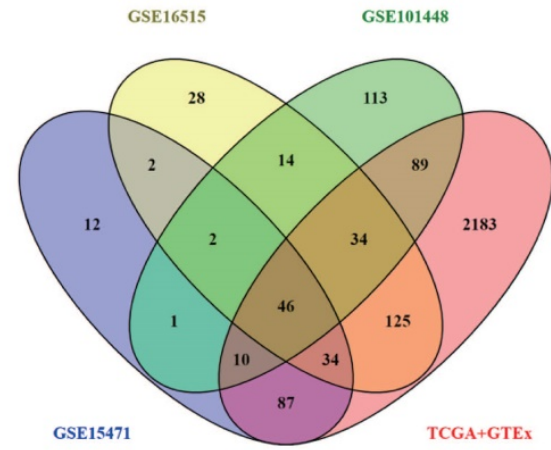

C

46 Common DEGs

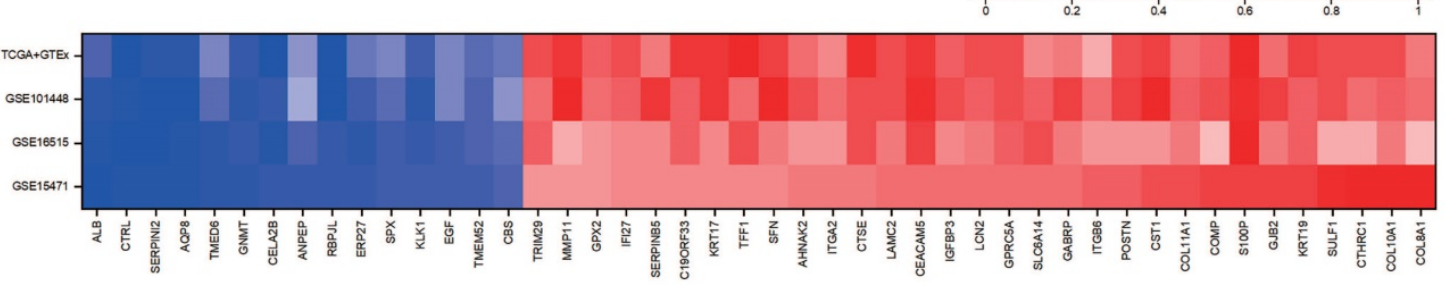

DEGs with log-rank P value $<0.05$ of both DFS and OS

\begin{tabular}{|ccc|}
\hline DEG & $\begin{array}{c}\text { DFS } \\
\text { (Logrank p value) }\end{array}$ & $\begin{array}{c}\text { OS } \\
\text { (Logrank } \text { p value) }\end{array}$ \\
\hline GPRC5A & $9.20 \mathrm{E}-05$ & 0.0026 \\
\hline C19ORF33 & 0.00029 & 0.00081 \\
\hline SERPINB5 & 0.00055 & $3.70 \mathrm{E}-05$ \\
\hline ITGB6 & 0.00092 & $1.70 \mathrm{E}-05$ \\
\hline TRIM29 & 0.00097 & 0.0032 \\
\hline ITGA2 & 0.0011 & 0.0037 \\
\hline LAMC2 & 0.0011 & 0.0018 \\
\hline S100P & 0.0039 & 0.0058 \\
\hline KRT19 & 0.0051 & $2.00 \mathrm{E}-04$ \\
\hline AHNAK2 & 0.0078 & 0.00012 \\
\hline KRT17 & 0.022 & 0.0091 \\
\hline GABRP & 0.026 & 0.0011 \\
\hline
\end{tabular}

E
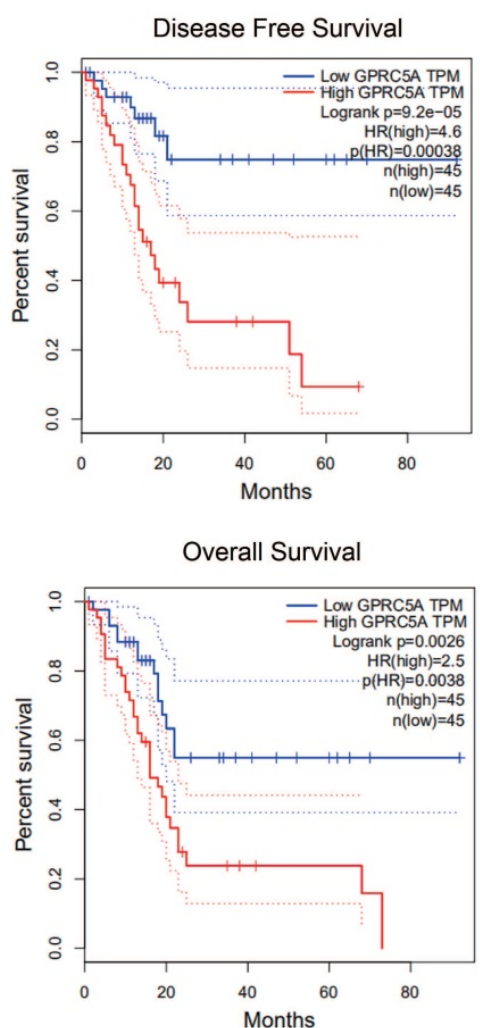

Figure 1. Identification of GPRC5A as a significant gene with prognostic value in PaCa. (A) Flow chart of selection. (B) 46 common DEGs between GSE15471, GSE16515, GSE101448 and TCGA plus GTEx datasets. (C) Log FC values of 46 common DEGs in GSE15471, GSE16515, GSE101448 and TCGA plus GTEx datasets. The red grids represent up-regulated genes and the blue grids represent down-regulated genes. (D) The genes with log rank $P$ value $<0.05$ of both DFS and OS. (E) The DFS and OS Kaplan-Meier curves of GPRC5A. The red curve represents GPRC5A high-expression group and the blue curve represents GPRC5A low-expression group. 
A

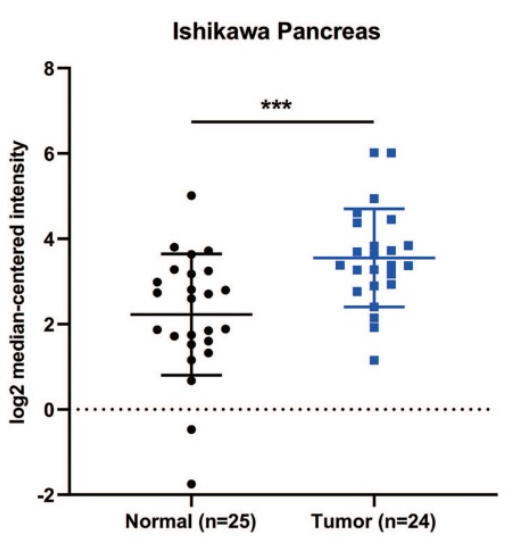

D

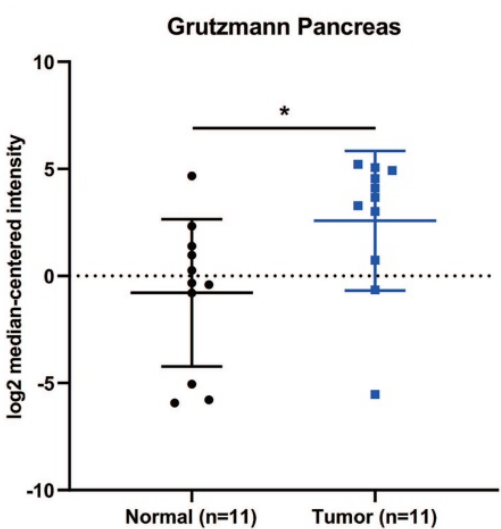

B

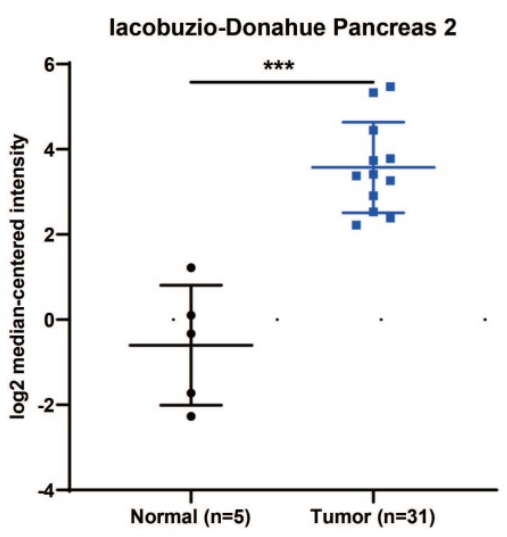

E

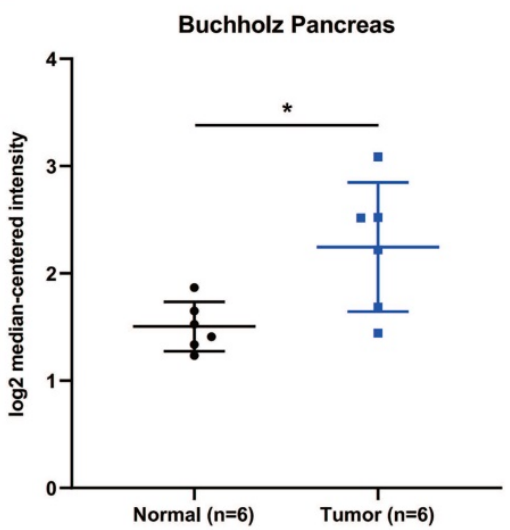

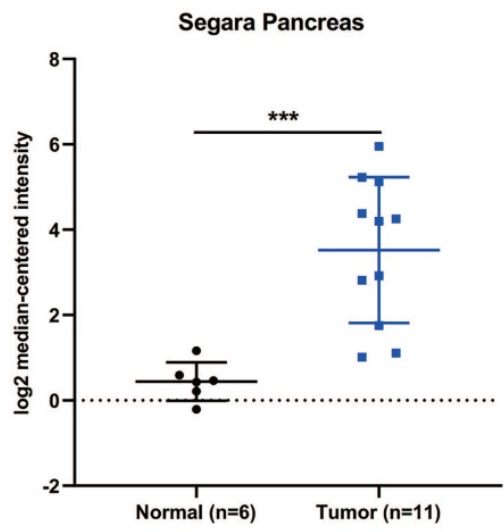

F

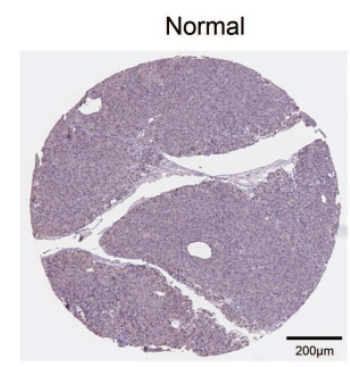

staining: Low

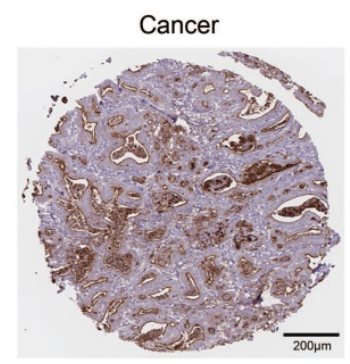

staining: High

GPRC5A

Figure 2. GPRC5A mRNA and protein expression were both significantly higher in PaCa than in normal pancreas tissues. (A-E) Scatter plots showing GPRC5A mRNA expression in, respectively, Ishikawa Pancreas (A), lacobuzio-Donahue Pancreas 2 (B), Segara Pancreas (C), Grutzmann Pancreas (D) and Buchholz Pancreas (E). (F) Representative immunohistochemistry images of GPRC5A in PaCa and normal pancreas tissues (The Human Protein Atlas), scale: $200 \mu \mathrm{m}$. Normal image URL: https://images.proteinatlas.org/7928/155465_A_2_3.jpg; Cancer image URL: https://www.proteinatlas.org/ENSG00000013588-GPRC5A/pathology/pancreatic+cancer\#timg.

\section{PaCa patients with mutant KRAS, TP53, CDKN2A or SMAD4 had higher expression of GPRC5A}

KRAS, TP53, CDKN2A and SMAD4 are the most common mutant genes in PaCa [7]. Regardless of mutation or non-mutation of the four genes, GPRC5A expression of tumor tissues was significantly higher than normal pancreas $(P<0.001)$. Of interest, in either mutant KRAS, TP53, CDKN2A or SMAD4, the expression of GPRC5A was much higher than the group with the corresponding non-mutation group
(Fig. 4A-D). The PaCa individuals with extremely low expression of GPRC5A, which was smaller than the median expression of normal group, oddly gathered in all non-mutation groups.

\section{Enrichment analyses of DEGs between GPRC5A high and low expression groups}

According to the expression of GPRC5A, we divided PaCa patients of TCGA cohort into high-expression and low-expression group. As demonstrated in Fig. 5A, 1806 genes were identified as DEGs between GPRC5A high and low expression 
groups, based on $|\log \mathrm{FC}|>1.0$ and $P<0.05$. Among them, 935 genes were up-regulated and 871 genes

A

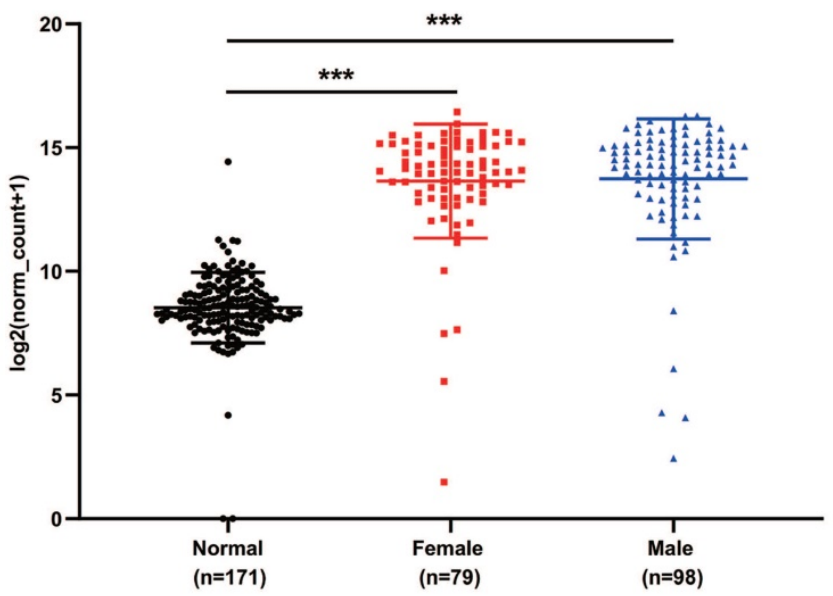

C

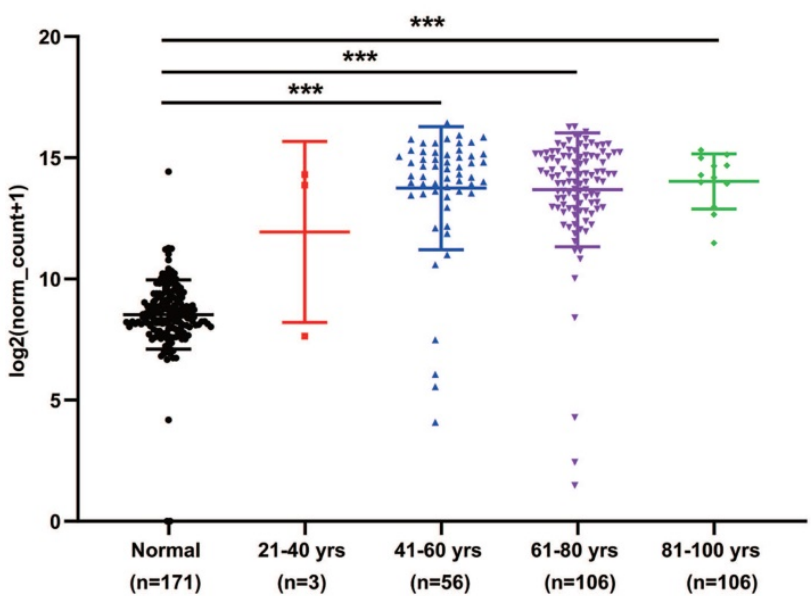

E

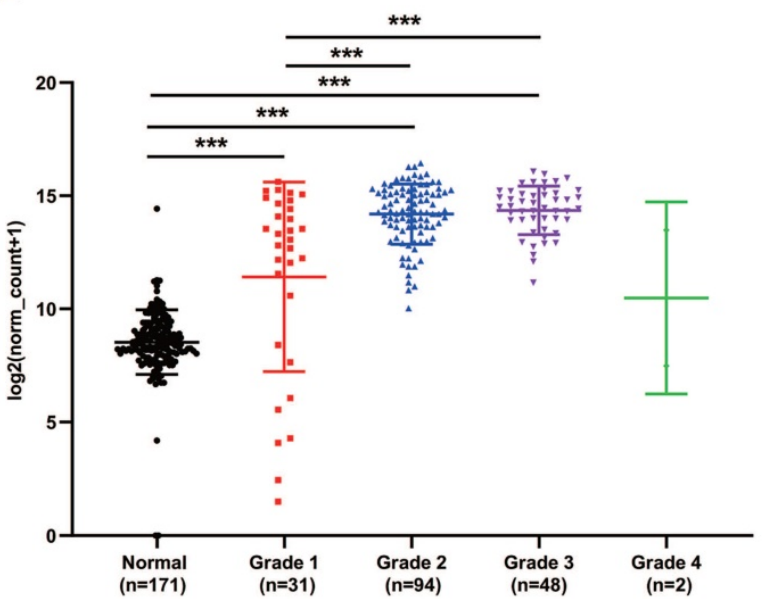

were down-regulated.

B

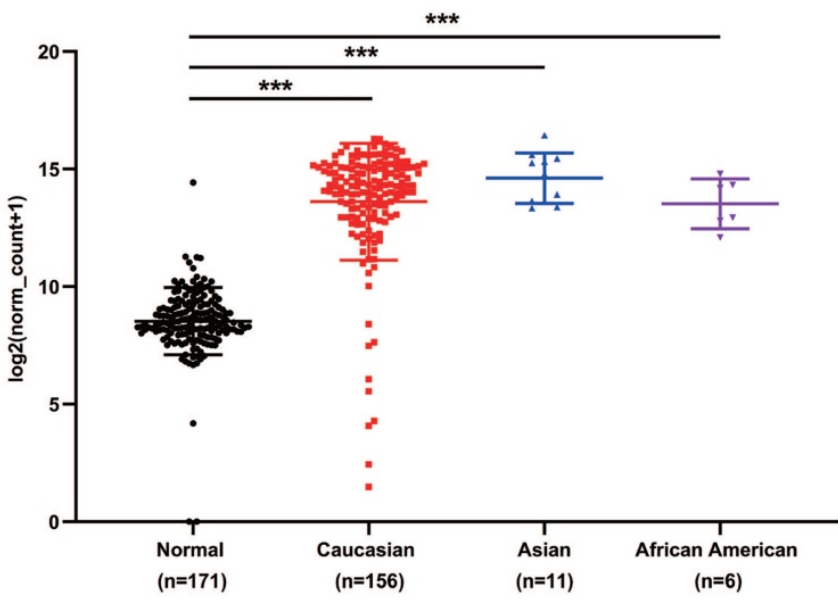

D

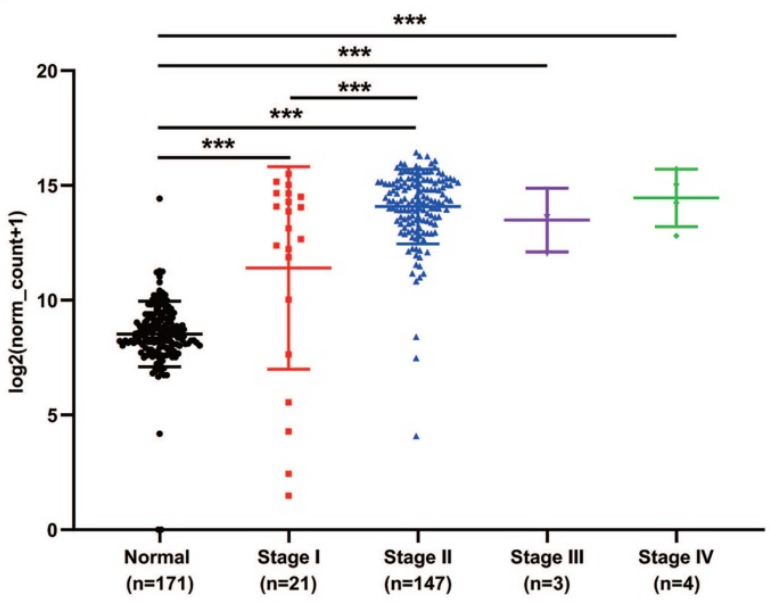

$\mathrm{F}$

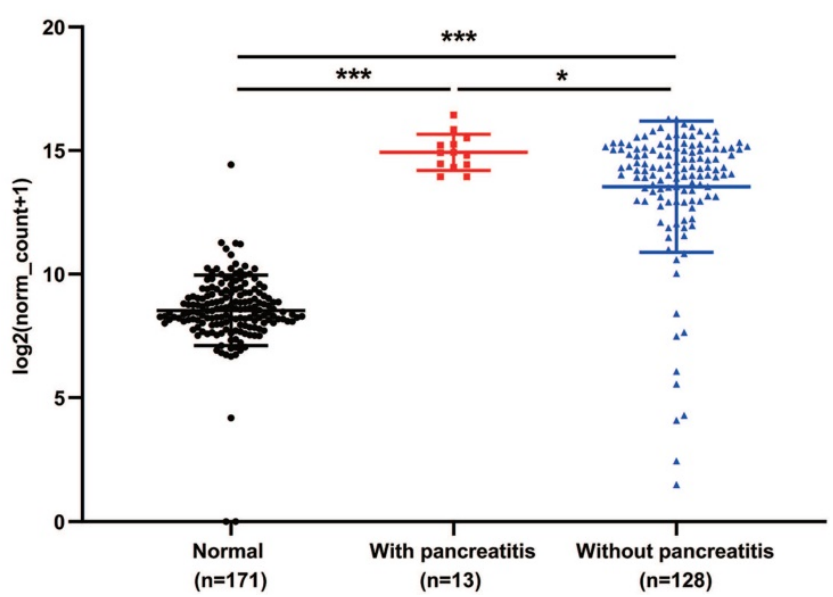

Figure 3. GPRC5A transcription in subgroups of patients with $\mathrm{PaCa}$, stratified based on gender, race, age and TNM stages, grade and with or without pancreatitis. (A) Scatter plot showing expression of GPRC5A in normal individuals of either gender or male or female PaCa patients. (B) Scatter plot showing expression of GPRC5A in normal individuals of any ethnicity or in PaCa patients of Caucasian, African-American or Asian ethnicity. (C) Scatter plot showing expression of GPRC5A in normal individuals of any age or in PaCa patients aged 21-40, 41-60, 61-80, or 8-100 yrs. (D) Scatter plot showing expression of GPRC5A in normal individuals or in PaCa patients in TNM stages 1, 2, 3 or 4. (E) Scatter plot showing expression of GPRC5A in normal individuals or PaCa patients with grade 1, 2, 3 or 4 tumors. (F) Scatter plot showing expression of GPRC5A in normal individuals or $\mathrm{PaCa}$ patients with or without pancreatitis. Data are mean $\pm \mathrm{SD}$. *, $P<0.05 ; * *, P<0.01 ; * * *, P<0.001$. 
A

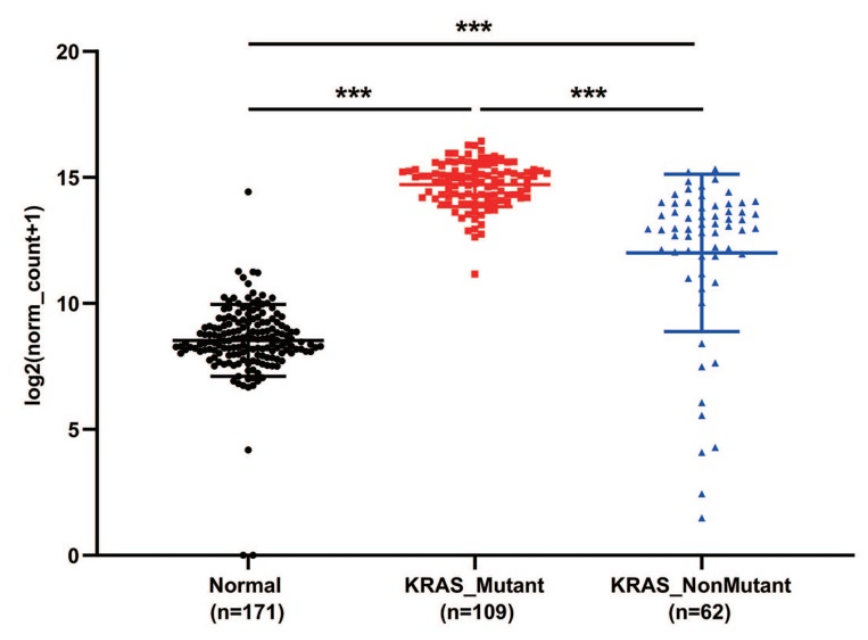

C

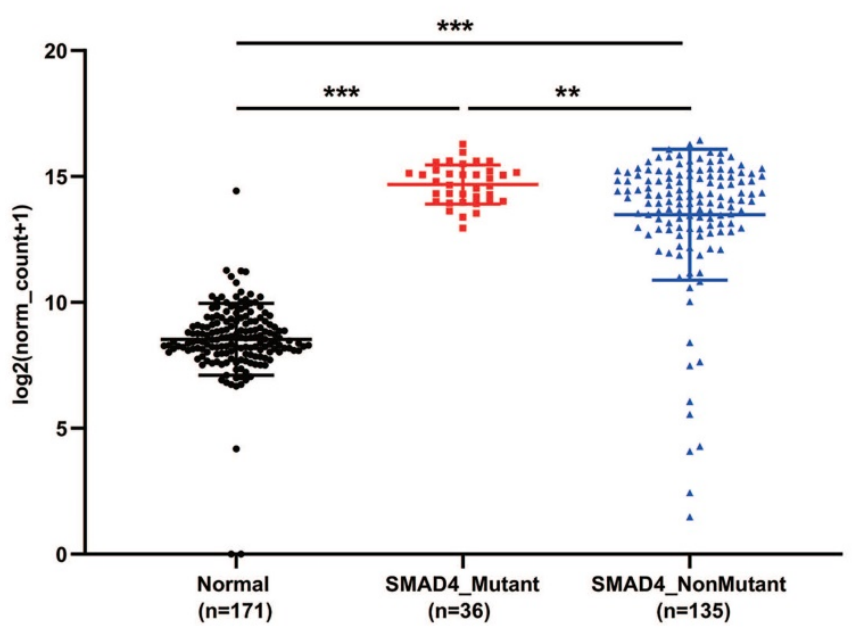

B

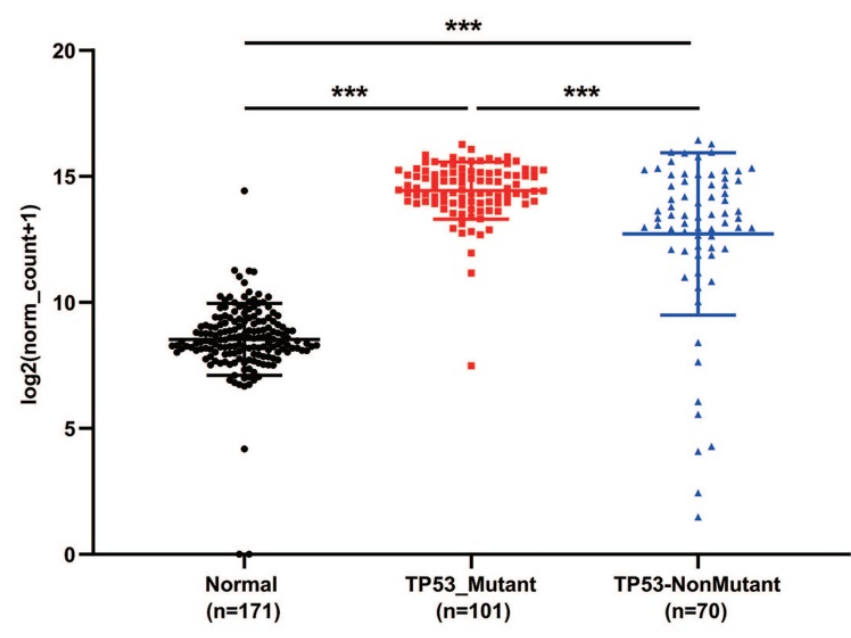

D

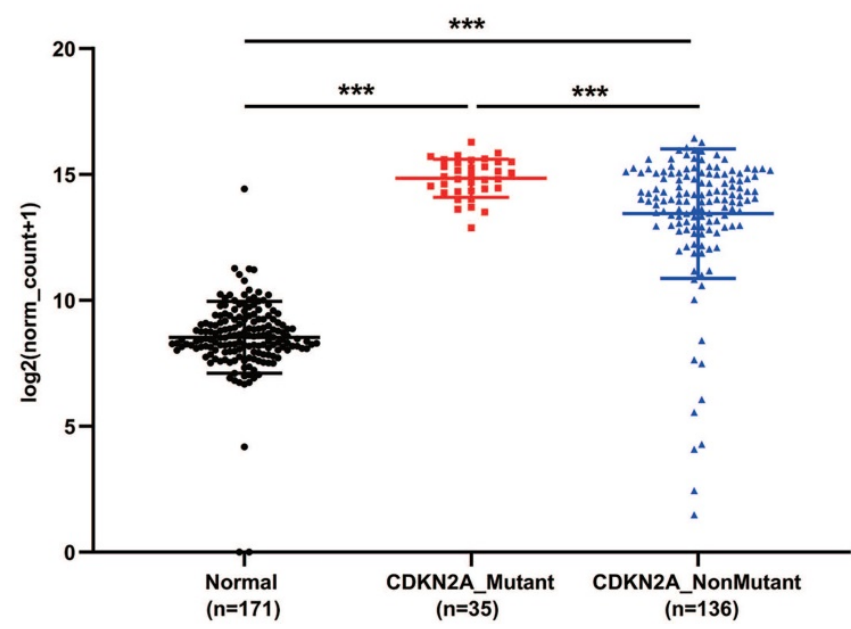

Figure 4. GPRC5A transcription in subgroups of patients with pancreatic cancer, stratified based on KRAS, TP53, SMAD4 and CDKN2A mutation. (A-D) Scatter plot showing expression of GPRC5A in normal individuals or PaCa patients with or without mutant KRAS (A), TP53 (B), SMAD4 (C) and CDKN2A (D). Data are mean \pm SD. $*, P<0.05 ; * *, P<0.01 ; * * *, P<0.001$.

GO, KEGG and Reactome enrichment analyses were performed by Metascape. Top 20 of the most enriched terms in the three datasets were listed respectively in Fig. 5B-D and detailed information was shown in Table S1-3. The DEGs were mainly enriched in ion channel activity, trans-synaptic signaling, presynapse, regulation of hormone levels and cell morphogenesis involved in differentiation by GO analysis (Fig. 5B). For the KEGG group, neuroactive ligand-receptor interaction, ECM-receptor interaction, calcium signaling pathway, retinol metabolism and serotonergic synapse were the most enriched pathways (Fig. 5C). Reactome analysis indicated that the DEGs were remarkably enriched in neuronal system, potassium channels, GPCR ligand binding, protein-protein interactions at synapses and hemostasis (Fig. 5D). Taken together, the gene sets related to neuronal system were the most mentioned and GPRC5A could play an important role in regulating nerve-related proteins.

The GSEA outcomes demonstrated that high GPRC5A expression was significantly correlated with metastasis, EMT, Semaphorin-4D (SEMA4D) in semaphorin signaling, SEMA4D induced cell migration and growth cone collapse and ephrin signaling (Fig. 5E). The other gene sets associated with tumor ranked in top 30 gene sets included hypoxia, tumor environment, NF-kB targets, RHO pathway, EGF response and epithelial differentiation (Fig. S1).

\section{Top 30 DEGs analyzed by GSEA between GPRC5A high and low expression groups}

A ranked gene list was generated by GSEA software. The top 15 genes with positive scores were GJB3, OSBPL3, LMO7, ASAP2, RHOF, JUP, KRT19, PITX1, LGALS3, MACC1, ACSL5, MAL2, VILL, ANXA2 and SFTA2. The top 15 genes with negative scores were SESN1, SMAD4, CDIP1, RFESD, SRR, 
ELAC1, HSF2, PRKN, HELQ, KLHL22, ZBTB40, RFXAP, SELENOP, MAP2K4 and ELP5. Gene full name, fold change, location and protein function were listed in Table 1.

\section{Clinical characteristics related to GPRC5A expression}

As demonstrated in Table 2, significant differences were observed between the highexpression and low-expression of GPRC5A patients regarding size of tumor $(\mathrm{T})$, TNM stage, grade $(\mathrm{G})$ and resection status (all $P<0.05$ ). On the aspect of size, 41 patients $(91.1 \%)$ with high-expression of GPRC5A were grouped into T3/4, whereas only $29(67.4 \%)$ patients were T3/4 in low-expression of GPRC5A group $(P=0.014)$. Furthermore, patients with high-expression of GPRC5A showed higher TNM stages $(P=0.015)$. In terms of grade, 40 patients $(88.9 \%)$ with high-expression of GPRC5A were in Grade 2/3, which displayed significantly poorer differentiation than GPRC5A low-expression group $(P=0.011)$. Besides, 17 patients (41.5\%) showed positive resection margin in GPRC5A high-expression group, while only 8 patients $(18.2 \%)$ were positive resection margin in low-expression group $(P=0.038)$. However, in terms of age, gender, lymph node and metastasis, no statistical differences were found between the two groups.

\section{Discussion}

Pancreatic cancer is highly aggressive and malignant, lacking efficacy molecular targets. Herein, we compared the gene expression of $\mathrm{PaCa}$ tissue and normal pancreas and identified 31 up-regulated DEGs and 15 down-regulated DEGs among four datasets. Further analysis revealed that 12 genes showed prognostic values, among which GPRC5A had the most significant prognostic value in DFS. The PaCa patients with higher expression of GPRC5A had a worse prognosis in both DFS and OS. In mutant KRAS, TP53, CDKN2A or SMAD4 group, GPRC5A expression was significantly higher than non-mutant group. Enrichment analysis revealed that GPRC5A could play a major role in metastasis of $\mathrm{PaCa}$ via regulating neuroactive ligand-receptor interaction and EMT. The analysis of clinical characteristics indicated that patients with high-expression of GPRC5A had larger tumor size, higher TNM stages, higher tumor grade, and more positive resection margin.

Table 1. Top 30 DEGs by GSEA analysis

\begin{tabular}{|c|c|c|c|c|}
\hline $\begin{array}{l}\text { Gene } \\
\text { symbol }\end{array}$ & Gene name & $\begin{array}{l}\text { Fold change; } \\
\text { GPRC5A, high/low }\end{array}$ & Location & Protein function \\
\hline SMAD4 & SMAD family member 4 & -0.91 & Nucleus/cytosol & Transcription factor \\
\hline RFXAP & Regulatory factor $X$ associated protein & -0.96 & Nucleus & Transcription factor \\
\hline HSF2 & Heat shock transcription factor 2 & -0.74 & Nucleus & Transcription factor \\
\hline ZBTB40 & Zinc finger and BTB domain containing 40 & -0.68 & Nucleus & Transcription factor \\
\hline PITX1 & Paired like homeodomain 1 & 4.43 & Nucleus & Transcription factor \\
\hline MACC1 & Metastasis-associated in colon cancer protein 1 & 2.04 & Nucleus/mitochondrion & Transcription activator \\
\hline MAP2K4 & Mitogen-activated protein kinase kinase 4 & -0.58 & Nucleus/cytosol & Kinase \\
\hline RHOF & Rho in filopodia & 2.65 & Extracellular/cytosol/cytoskeleton & GTPase \\
\hline SESN1 & Sestrin 1 & -1.21 & Nucleus/cytosol & Enzyme \\
\hline PRKN & Parkin RBR E3 ubiquitin protein ligase & -1.16 & Nucleus/cytosol/mitochondrion & Enzyme \\
\hline ELAC1 & ElaC ribonuclease $\mathrm{Z} 1$ & -1.01 & Nucleus/cytosol & Enzyme \\
\hline SRR & Serine racemase & -0.87 & Cytosol & Enzyme \\
\hline HELQ & Helicase POLQ like & -0.51 & Nucleus & Enzyme \\
\hline ACSL5 & Acyl-CoA synthetase long chain family member 5 & 2.12 & Membrane/nucleus/mitochondrion & Enzyme \\
\hline GJB3 & Gap Junction Protein Beta 3 & 4.43 & Membrane & Transporter \\
\hline ANXA2 & Annexin A2 & 1.61 & Membrane/nucleus/extracellular & Transporter \\
\hline CDIP1 & Cell death inducing p53 target 1 & -1.24 & Endosome/lysosome/membrane & Other \\
\hline KLHL22 & Kelch like family member 22 & -0.86 & Cytosol & Other \\
\hline RFESD & Rieske domain-containing protein & -0.83 & Unknown & Other \\
\hline ELP5 & Elongator acetyltransferase complex subunit 5 & -0.71 & Nucleus/cytosol & Other \\
\hline ASAP2 & ArfGAP with $\mathrm{SH} 3$ domain, ankyrin repeat and $\mathrm{PH}$ domain 2 & 1.37 & Membrane/golgi apparatus & Other \\
\hline JUP & Junction plakoglobin & 1.53 & Membrane/nucleus & Other \\
\hline LMO7 & LIM domain 7 & 1.54 & Nucleus/cytosol & Other \\
\hline OSBPL3 & Oxysterol binding protein like 3 & 1.64 & Nucleus/cytosol & Other \\
\hline KRT19 & Keratin 19 & 2.89 & Extracellular/cytosol/cytoskeleton & Other \\
\hline LGALS3 & Galectin 3 & 1.83 & Membrane/extracellular/nucleus & Other \\
\hline MAL2 & Mal, $\mathrm{T}$ cell differentiation protein 2 & 1.97 & Membrane & Other \\
\hline VILL & Villin like & 2.66 & Cytosol/cytoskeleton & Other \\
\hline SFTA2 & Surfactant associated 2 & 5.12 & Extracellular/golgi apparatus & Other \\
\hline SELENOP & Selenoprotein $\mathrm{P}$ & -1.31 & Extracellular & Other \\
\hline
\end{tabular}


A TCGA PAAD RNA Seq (GPRC5A High/Low)

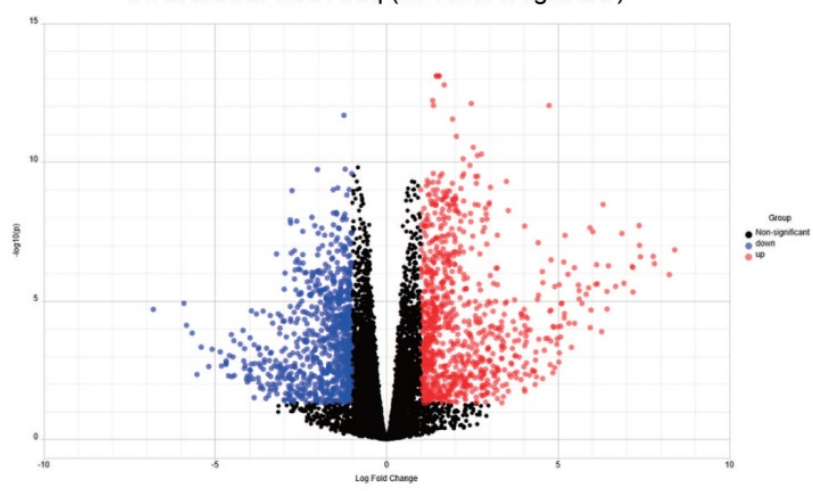

B

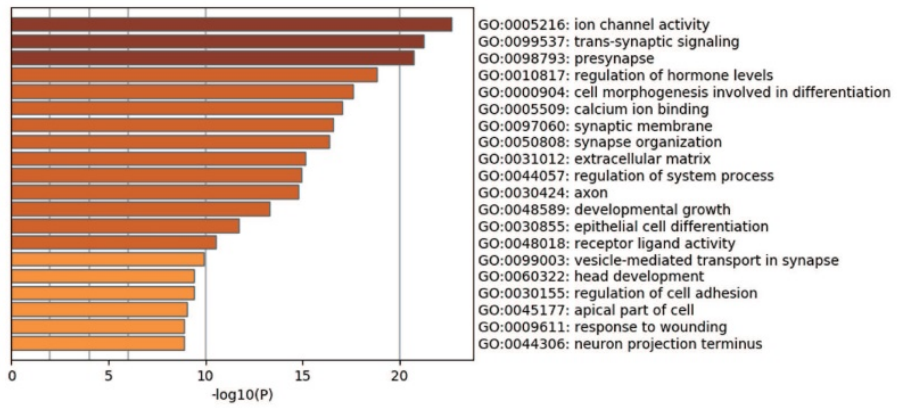

C

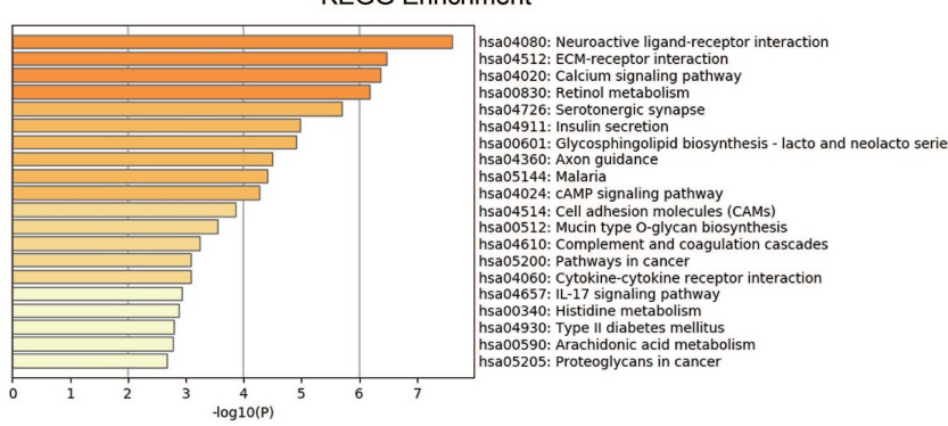

D

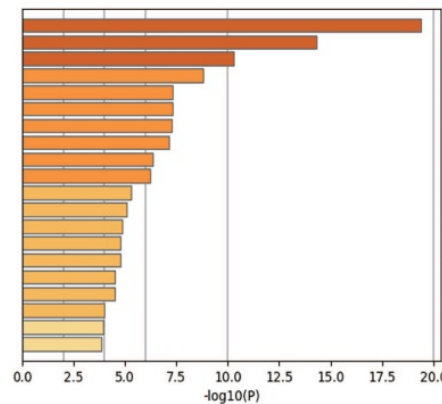

E

10 of top 30 gene sets enriched in GPRC5A high-expression group:

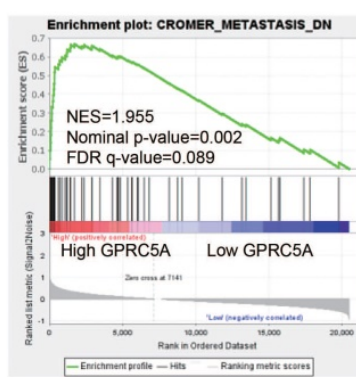

Enrichment plot: JAEGER_METASTASIS_DN

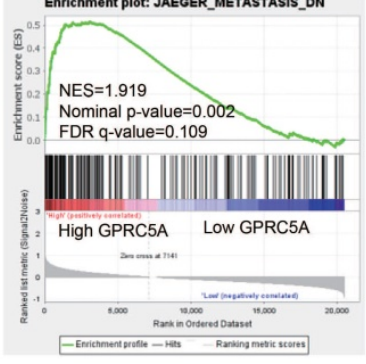

Enrichment plot:
PID_ECADHERIN_STABILIZATION_PATHWAY
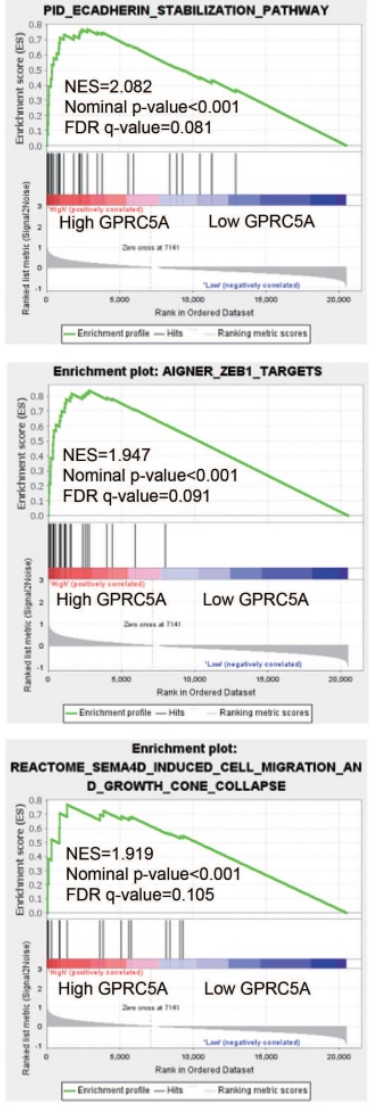

Enrichment plot: RICKMAN_METASTASIS_DN

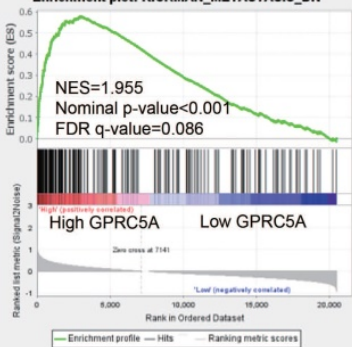

Enrichment plot: ONDER_CDH1_TARGETS_2_DN

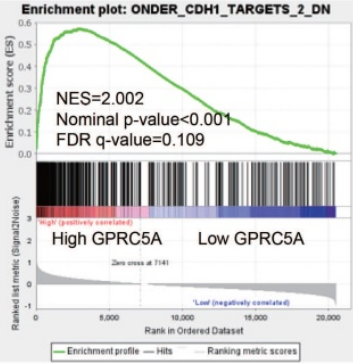

Enrichment plot: HOLLERN_EMT_BREAST_TUMOR_DN

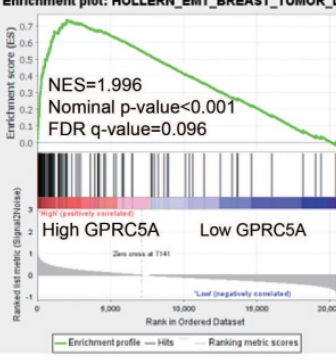

Enrichment plot:

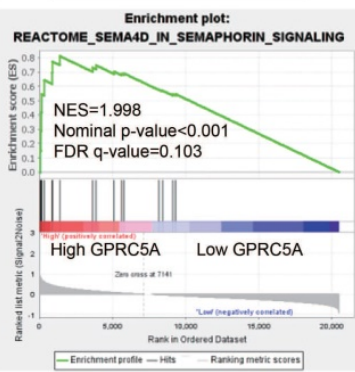

Enrichment plot: REACTOME_EPH_EPHRIN_SIGNAUINO

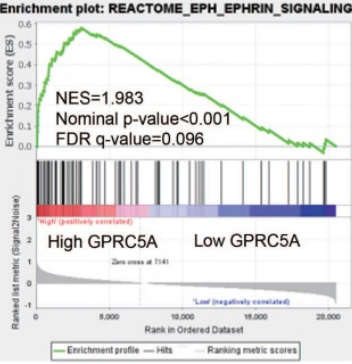

Figure 5. Identification of DEGs between GPRC5A high-expression and low-expression groups, and enrichment analyses of DEGs. (A) Volcano plot of all DEGs between GPRC5A high-expression and low-expression group. Up-regulated genes, down-regulated genes and unchanged genes were marked as red, blue and gray dots, respectively. (B) Top 20 terms of GO enrichment analysis of DEGs. (C) Top 20 pathways of KEGG enrichment analysis of DEGs. (D) Top 20 terms of enrichment analysis in Reactome Gene Sets. (E) 10 of top 30 gene sets enriched in GPRC5A high-expression group analyzed by GSEA.

\section{GPRC5A acted as an oncogene in PaCa}

$\mathrm{G}$ protein-coupled receptor class $\mathrm{C}$ group 5 member A (GPRC5A), also known as retinoic acid-induced gene 3 (RAI3) or retinoic acid-induced gene 1 (RAIG1) is a member of class C orphan GPCRs, located on the cell membrane and endoplasmic reticulum $[33,37]$. The current studies confirm that 
GPRC5A has a retinoic acid response element (RARE) in its $5^{\prime}$ upstream region and the transcription of the gene can be induced by retinoic acid [38, 39]. In addition, cAMP, TP53, BRCA1 and hypoxia-inducible factors (HIFs) were reported to regulate the transcription of GPRC5A [40-43]. The expression of GPRC5A differed in various human cancers, indicating its dual role in tumorigenesis. In colorectal cancer study, GPRC5A was induced by hypoxia and was regulated major by HIFs. The downstream activation of YAP by hypoxia required GPRC5A, which enabled hypoxic cell survival by suppressing apoptosis via BCL-XL induction [40]. Studies in gastric cancer also suggested that GPRC5A was an oncogene exerting its function by regulating EMT or the epidermal growth factor receptor (EGFR) signaling [44, 45]. Sawada et al. reported that in prostate cancer GPRC5A facilitated cell proliferation through cell cycle regulation and was significantly essential for bone metastasis [46]. Besides, in ovarian cancer, it was shown that the adaptive ERK1/ 2-RSK1/2-EphA2-GPRC5A signaling switch triggered chemotherapy resistance and identified GPRC5A as a marker for abysmal ovarian cancer outcome [47]. In contrast, GPRC5A is generally recognized as a tumor suppressor gene in lung cancer [48]. Deletion of Gprc5a resulted in persistent signal transducer and activator of transcription 3 (Stat3) activation that was important for lung cancer cell survival and transformation [49].

In the present study, we identified that in both mRNA and protein level, GPRC5A was lowly expressed in normal pancreas while significantly up-regulated in PaCa tissues. We also found that high-level GPRC5A usually predicted significantly shorter OS and DFS (see Fig. 1). As shown in Table 2, high expression of GPRC5A was associated with worse clinical characteristics among $\mathrm{PaCa}$ patients. Recently, Zhou et al. reported that GPRC5A promoted $\mathrm{PaCa}$ cell growth and migration, and enhanced resistance to gemcitabine by regulating human antigen $\mathrm{R}$ (HuR), which was a key mediator of gemcitabine's efficacy in cancer cells [36]. Liu et al. verified the cancer-promoting role of GPRC5A in $\mathrm{PaCa}$ and found that when GPRC5A was knocked out, the inactivated phosphorylation of GSK-3 $\beta$ (Glycogen synthase kinase-3 $\beta$ ) was upregulated [35]. Jahny et al. also found that GPRC5A activated STAT3 in $\mathrm{PaCa}$, which was discrepancy with its function on STAT3 in lung cancer [34]. The function of GPRC5A in cancers may need to be discussed under specific context of tumor. To sum up, GPRC5A acted as an oncogene in PaCa.
Table 2. Clinical characteristics and comparisons of two pancreatic cancer cohorts

\begin{tabular}{|c|c|c|c|c|}
\hline & $\begin{array}{l}\text { GPRC5A, } \\
\text { Low }(n=45)\end{array}$ & $\begin{array}{l}\text { GPRC5A, High } \\
(\mathrm{n}=45)\end{array}$ & $P$ value & Total \\
\hline Age (years, median) & 65 & 65 & $0.486^{*}$ & - \\
\hline \multicolumn{5}{|l|}{ Gender } \\
\hline Male & 24 & 27 & \multirow[t]{2}{*}{$0.523^{\#}$} & 51 \\
\hline Female & 21 & 18 & & 39 \\
\hline \multicolumn{5}{|l|}{ Size of Tumor (T) } \\
\hline $\mathrm{T} 1$ & 3 & 1 & \multirow[t]{6}{*}{$0.014^{a}$} & 4 \\
\hline T2 & 11 & 3 & & 14 \\
\hline T3 & 28 & 41 & & 69 \\
\hline $\mathrm{T} 4$ & 1 & 0 & & 1 \\
\hline Unknown & 2 & 0 & & 2 \\
\hline \multicolumn{4}{|l|}{ Lymph Node (N) } & \\
\hline No & 15 & 9 & \multirow[t]{3}{*}{$0.130^{\#}$} & 24 \\
\hline N1 & 27 & 34 & & 61 \\
\hline Unknown & 3 & 2 & & 5 \\
\hline \multicolumn{5}{|l|}{ Metastasis (M) } \\
\hline M0 & 18 & 22 & \multirow[t]{4}{*}{$1.000^{a}$} & 40 \\
\hline M1 & 1 & 2 & & 3 \\
\hline Unknown & 26 & 21 & & 47 \\
\hline \multicolumn{4}{|l|}{ AJCC TNM Stage } & \\
\hline I & 10 & 2 & \multirow[t]{6}{*}{$0.015^{a}$} & 12 \\
\hline II & 31 & 41 & & 72 \\
\hline III & 1 & 0 & & 1 \\
\hline IV & 1 & 2 & & 3 \\
\hline Unknown & 2 & 0 & & 2 \\
\hline \multicolumn{4}{|l|}{ Grade (G) } & \\
\hline G1 & 16 & 5 & \multirow[t]{5}{*}{$0.011^{a}$} & 21 \\
\hline G2 & 17 & 28 & & 45 \\
\hline G3 & 9 & 12 & & 21 \\
\hline G4 & 1 & 0 & & 1 \\
\hline Unknown & 2 & 0 & & 2 \\
\hline \multicolumn{5}{|l|}{ Resection status } \\
\hline R0 & 36 & 24 & \multirow[t]{4}{*}{$0.038^{a}$} & 60 \\
\hline R1 & 7 & 16 & & 23 \\
\hline R2 & 1 & 1 & & 2 \\
\hline Unknown & 1 & 4 & & 5 \\
\hline
\end{tabular}

*Unpaired $\mathrm{t}$ test; "X2 Test; $a$ Fisher's exact test.

\section{GPRC5A collaborated with driver genes in $\mathrm{PaCa}$}

In the present, compared with non-mutant group, GPRC5A was expressed higher in the patients with mutant KRAS, TP53, CDKN2A or SMAD4, which are the four key diver genes in PaCa [8].

Previous studies identified that in most tumor cell lines expressing mutant p53, the expression of GPRC5A was elevated, whereas it was relatively repressed in the tumor cell lines expressing wild-type p53 [43]. p53 interacted with the promoter of GPRC5A and repressed its expression at the onset of apoptosis [43]. Recently, it was reported that Gprc5a-/- mice spontaneously developed lung adenocarcinomas with or without chronically nicotine-specific carcinogen exposure. Their mutational landscape exhibited markedly high somatic mutation burdens in the Kras oncogene $[50,51]$. It was also reported that depletion of Gprc5a activated TGF- $\beta$ signaling in podocytes [52]. So far, studies about the correlation between GPRC5A and the driver genes are still limited. In our analysis, 
higher expression of GPRC5A was shown in the mutant group, which indicated that GPRC5A collaborated with key driver genes in PaCa. More studies are needed to clarify the natural order and causal relationship between GPRC5A and the four mutant genes.

A number of drugs targeting GPCRs have been successfully designed, with a market composition of $20 \%-30 \%$ [53]. Considering the role of GPRC5A as a member of GPCR of receptor class C, it has the potential to be a new target for drug discovery, although there is still no evidence supporting that it can be ligand activated [54]. Up to date, studies on GPRC5A are still limited. However, with the development of screening technologies, the extreme abnormal expression of GPRC5A has been noticed in different cancers. Thus, its signaling function and identification of ligands deserve more nuanced exploration.

\section{GPRC5A may regulate neuroactive ligand-receptor interaction and EMT}

In the study, the GO, KEGG and Reactome enrichment of DEGs focused on trans-synaptic signaling, pre-synapse, neuroactive ligand-receptor interaction and neural system, respectively. The results of GSEA also enriched in the gene sets related to axon guidance-associated signaling, i.e., Ephrin and SEMA4D signaling. Up to $100 \%$ of PaCa patients had perineural invasion [55-57]. Neural plasticity is an intrinsic characteristic of pancreatic cancer, which involves neuronal activation at different levels in the nerve $[58,59]$. The severity of neural invasion was shown to be an independent prognostic factor for OS in $\mathrm{PaCa}[58,60]$. Recent studies also demonstrated that ephrin and SEMA4D signaling promoted malignancy of PaCa [61-63]. GPRC5A was reported to directly interact with ephrin type-A receptor 2 (EphA2), a receptor tyrosine kinase (RTK) which played a key role in epithelial tumor metastases and invasiveness $[47,64]$.

On the other hand, SEMA4D is expected to be a new target for treating PaCa $[62,63]$. Phase I clinical trial of a humanized anti-Sema4D antibody VX15/2503 (Vaccinex) has been shown to prolong 8 weeks of progression-free survival [62]. Based on bioinformatics analysis, we first raised the possibility of the correlation between the signaling modulated by the two genes. The correlation could be expected. Recent studies showed that SEMA4D mediated activation of transforming protein RhoA (RHOA) and activation of RHOA could upregulate GPRC5A [65, 66]. The relation could be linked by Rho GTPases; however, it needs more studies to verify.

Apart from pathways related to neuroactive ligand-receptor interactions, GESA results also showed that high-expression of GPRC5A was significantly associated with the gene sets of metastasis and EMT, which indicated that GPRC5A might play an important role in promoting metastasis (see in Fig. 5). Studies on GPRC5A regulating EMT are few. However, an explanation could be the activation of STAT3 mediated by TGF- $\beta$ in cooperation with active K-Ras inducing EMT [67]. In addition, active YAP and inactive GSK-3 $\beta$ may also mediate EMT [68, 69]. Moreover, we also listed the other ten important gene sets enriched in high-expression group (Fig. S1). It seemed that GPRC5A could affect signaling related to hypoxia, tumor environment, Rho GTPase, NF-kB and EGF response. These enrichment results were in accordance with the clinical outcomes and supported that GPRC5A acted as an oncogene in PaCa.

\section{Limitation of the study}

The limitation of the study was the unequal distribution of the patients enrolled in our analysis. The sample size of the group of 21-40 yrs was small, which influenced the statistical comparison. Since the patients included were gathered in Stage I and II, the numbers of patients included in Stage III and IV, or in grade 4 group, were quite small. Therefore, more samples distributed equally are needed to be involved to validate the results.

\section{Conclusion}

Our study presented an overview of GPRC5A in PaCa by utilizing and analyzing the public datasets. The results strongly suggested that GPRC5A could exert pro-malignancy in PaCa. Besides, we also offered some new target genes and pathways related to GPRC5A, which deserved further exploration.

\section{Abbreviations}

PaCa: pancreatic cancer; DEGs: differentially expressed genes; GEO: Gene Expression Omnibus; GSEA: gene set enrichment analysis; TCGA: The Cancer Genome Atlas; GPRC5A: G protein-coupled receptor class $C$ group 5 member $A$; OS: overall survival; DFS: disease-free survival; GTEx: Genotype Tissue Expression; GO: Gene Ontology; KEGG: Kyoto Encyclopedia of Genes and Genomes; EMT: epithelialmesenchymal transition; GPCRs: G protein-coupled receptors; IHC: immunohistochemical; TNM: Tumor Node Metastasis; RTK: receptor tyrosine kinase.

\section{Supplementary Material}

Supplementary figures and tables. http://www.jcancer.org/v12p2010s1.pdf 


\section{Acknowledgements}

We would like to thank Dr. Chang Cui from the First Affiliated Hospital of Nanjing Medical University for offering scientific suggestions. The study was funded by the National Natural Science Foundation of China (81871947).

\section{Author contributions}

Conception and design: XZ, SS. Development of methodology: $X Q$. Acquisition of data: $X Q, C J$. Analysis and interpretation of data: XQ. Writing of the manuscript: $X Q$. Administrative, technical, or material support: $X Q, C J$. Study supervision: $X Z$, SS.

\section{Competing Interests}

The authors have declared that no competing interest exists.

\section{References}

1. Siegel RL, Miller KD, Jemal A. Cancer statistics, 2019. CA Cancer J Clin. 2019; 69: 7-34

2. Rahib L, Smith BD, Aizenberg R, Rosenzweig AB, Fleshman JM, Matrisian LM. Projecting cancer incidence and deaths to 2030: the unexpected burden of thyroid, liver, and pancreas cancers in the United States. Cancer Res. 2014; 74: 2913-21.

3. Murakawa M, Aoyama T, Miyagi Y, Kobayashi S, Ueno M, Morimoto M, et al. The impact of SPARC expression on the survival of pancreatic ductal adenocarcinoma patients after curative resection. J Cancer. 2019; 10: 627-33.

4. Kamisawa T, Wood LD, Itoi T, Takaori K. Pancreatic cancer. Lancet. 2016; 388 : 73-85.

5. Demir IE, Friess H, Ceyhan GO. Neural plasticity in pancreatitis and pancreatic cancer. Nat Rev Gastroenterol Hepatol. 2015; 12: 649-59.

6. Jiang H, Hegde S, DeNardo DG. Tumor-associated fibrosis as a regulator of tumor immunity and response to immunotherapy. Cancer Immunol Immunother. 2017; 66: 1037-48.

7. Bailey P, Chang DK, Nones K, Johns AL, Patch AM, Gingras MC, et al. Genomic analyses identify molecular subtypes of pancreatic cancer. Nature. 2016; 531: 47-52.

8. Collisson EA, Sadanandam A, Olson P, Gibb WJ, Truitt M, Gu S, et al. Subtypes of pancreatic ductal adenocarcinoma and their differing responses to therapy. Nat Med. 2011; 17: 500-3.

9. Badea L, Herlea V, Dima SO, Dumitrascu T, Popescu I. Combined gene expression analysis of whole-tissue and microdissected pancreatic ductal adenocarcinoma identifies genes specifically overexpressed in tumor epithelia. Hepatogastroenterology. 2008; 55: 2016-27.

10. Barrett T, Wilhite SE, Ledoux P, Evangelista C, Kim IF, Tomashevsky M, et al. NCBI GEO: archive for functional genomics data sets--update. Nucleic Acids Res. 2013; 41: D991-5.

11. Edgar R, Domrachev M, Lash AE. Gene Expression Omnibus: NCBI gene expression and hybridization array data repository. Nucleic Acids Res. 2002; 30: 207-10.

12. Ellsworth KA, Eckloff BW, Li L, Moon I, Fridley BL, Jenkins GD, et al. Contribution of FKBP5 genetic variation to gemcitabine treatment and survival in pancreatic adenocarcinoma. PLoS One. 2013; 8: e70216.

13. Idichi T, Seki N, Kurahara H, Yonemori K, Osako Y, Arai T, et al. Regulation of actin-binding protein ANLN by antitumor miR-217 inhibits cancer cell aggressiveness in pancreatic ductal adenocarcinoma. Oncotarget. 2017; 8: 53180-93.

14. Klett H, Fuellgraf H, Levit-Zerdoun E, Hussung S, Kowar S, Kusters S, et al. Identification and Validation of a Diagnostic and Prognostic Multi-Gene Biomarker Panel for Pancreatic Ductal Adenocarcinoma. Front Genet. 2018; 9: 108.

15. Li L, Zhang JW, Jenkins G, Xie F, Carlson EE, Fridley BL, et al. Genetic variations associated with gemcitabine treatment outcome in pancreatic cancer. Pharmacogenet Genomics. 2016; 26: 527-37.

16. Pei H, Li L, Fridley BL, Jenkins GD, Kalari KR, Lingle W, et al. FKBP51 affects cancer cell response to chemotherapy by negatively regulating Akt. Cancer Cell. 2009; 16: 259-66.

17. Tang Z, Li C, Kang B, Gao G, Li C, Zhang Z. GEPIA: a web server for cancer and normal gene expression profiling and interactive analyses. Nucleic Acids Res. 2017; 45: W98-W102.

18. Xia J, Benner MJ, Hancock RE. NetworkAnalyst--integrative approaches for protein-protein interaction network analysis and visual exploration. Nucleic Acids Res. 2014; 42: W167-74.
19. Xia J, Gill EE, Hancock RE. NetworkAnalyst for statistical, visual and network-based meta-analysis of gene expression data. Nat Protoc. 2015; 10: 823-44.

20. Xia J, Fjell CD, Mayer ML, Pena OM, Wishart DS, Hancock RE. INMEX--a web-based tool for integrative meta-analysis of expression data. Nucleic Acids Res. 2013; 41: W63-70.

21. Xia J, Lyle NH, Mayer ML, Pena OM, Hancock RE. INVEX--a web-based tool for integrative visualization of expression data. Bioinformatics. 2013; 29: 3232-4.

22. Zhou G, Soufan O, Ewald J, Hancock REW, Basu N, Xia J. NetworkAnalyst 3.0: a visual analytics platform for comprehensive gene expression profiling and meta-analysis. Nucleic Acids Res. 2019; 47: W234-W41.

23. Buchholz M, Braun M, Heidenblut A, Kestler HA, Kloppel G, Schmiegel W, et al. Transcriptome analysis of microdissected pancreatic intraepithelial neoplastic lesions. Oncogene. 2005; 24: 6626-36.

24. Grutzmann R, Pilarsky C, Ammerpohl O, Luttges J, Bohme A, Sipos B, et al. Gene expression profiling of microdissected pancreatic ductal carcinomas using high-density DNA microarrays. Neoplasia. 2004; 6: 611-22.

25. Iacobuzio-Donahue CA, Maitra A, Olsen M, Lowe AW, van Heek NT, Rosty C, et al. Exploration of global gene expression patterns in pancreatic adenocarcinoma using cDNA microarrays. Am J Pathol. 2003; 162: 1151-62.

26. Ishikawa M, Yoshida K, Yamashita Y, Ota J, Takada S, Kisanuki H, et al. Experimental trial for diagnosis of pancreatic ductal carcinoma based on gene expression profiles of pancreatic ductal cells. Cancer Sci. 2005; 96: 387-93.

27. Rhodes DR, Yu J, Shanker K, Deshpande N, Varambally R, Ghosh D, et al. ONCOMINE: a cancer microarray database and integrated data-mining platform. Neoplasia. 2004; 6: 1-6.

28. Segara D, Biankin AV, Kench JG, Langusch CC, Dawson AC, Skalicky DA, et al. Expression of HOXB2, a retinoic acid signaling target in pancreatic cancer and pancreatic intraepithelial neoplasia. Clin Cancer Res. 2005; 11: 3587-96.

29. Uhlen M, Fagerberg L, Hallstrom BM, Lindskog C, Oksvold P, Mardinoglu A, et al. Proteomics. Tissue-based map of the human proteome. Science. 2015; 347: 1260419.

30. Goldman MJ, Craft B, Hastie M, Repecka K, McDade F, Kamath A, et al. Visualizing and interpreting cancer genomics data via the Xena platform. Nat Biotechnol. 2020; 38: 675-8

31. Zhou Y, Zhou B, Pache L, Chang M, Khodabakhshi AH, Tanaseichuk O, et al. Metascape provides a biologist-oriented resource for the analysis of systems-level datasets. Nat Commun. 2019; 10: 1523.

32. Subramanian A, Tamayo P, Mootha VK, Mukherjee S, Ebert BL, Gillette MA, et al. Gene set enrichment analysis: a knowledge-based approach for interpreting genome-wide expression profiles. Proc Natl Acad Sci U S A. 2005; 102: $15545-50$

33. Wang J, Farris $\mathrm{AB}, \mathrm{Xu} \mathrm{K}$, Wang $\mathrm{P}$, Zhang $\mathrm{X}$, Duong DM, et al. GPRC5A suppresses protein synthesis at the endoplasmic reticulum to prevent radiation-induced lung tumorigenesis. Nat Commun. 2016; 7: 11795.

34. Jahny E, Yang H, Liu B, Jahnke B, Lademann F, Knosel T, et al. The G Protein-Coupled Receptor RAI3 Is an Independent Prognostic Factor for Pancreatic Cancer Survival and Regulates Proliferation via STAT3 Phosphorylation. PLoS One. 2017; 12: e0170390.

35. Liu B, Yang H, Pilarsky C, Weber GF. The Effect of GPRC5a on the Proliferation, Migration Ability, Chemotherapy Resistance, and Phosphorylation of GSK-3beta in Pancreatic Cancer. Int J Mol Sci. 2018; 19: 1870.

36. Zhou $\mathrm{H}$, Telonis AG, Jing $\mathrm{Y}$, Xia NL, Biederman $\mathrm{L}$, Jimbo $\mathrm{M}$, et al. GPRC5A is a potential oncogene in pancreatic ductal adenocarcinoma cells that is upregulated by gemcitabine with help from HuR. Cell Death Dis. 2016; 7: e2294

37. Cheng Y, Lotan R. Molecular cloning and characterization of a novel retinoic acid-inducible gene that encodes a putative $G$ protein-coupled receptor. J Biol Chem. 1998; 273: 35008-15.

38. Allenby G, Bocquel MT, Saunders M, Kazmer S, Speck J, Rosenberger M, et al. Retinoic acid receptors and retinoid $X$ receptors: interactions with endogenous retinoic acids. Proc Natl Acad Sci U S A. 1993; 90: 30-4.

39. Ye $\mathrm{X}$, Tao $\mathrm{O}$, Wang $\mathrm{Y}$, Cheng $\mathrm{Y}$, Lotan $\mathrm{R}$. Mechanisms underlying the induction of the putative human tumor suppressor GPRC5A by retinoic acid. Cancer Biol Ther. 2009; 8: 951-62.

40. Greenhough A, Bagley C, Heesom KJ, Gurevich DB, Gay D, Bond M, et al. Cancer cell adaptation to hypoxia involves a HIF-GPRC5A-YAP axis. EMBO Mol Med. 2018; 10: e8699.

41. Hirano M, Zang L, Oka T, Ito $Y$, Shimada $Y$, Nishimura $Y$, et al. Novel reciprocal regulation of CAMP signaling and apoptosis by orphan G-protein-coupled receptor GPRC5A gene expression. Biochem Biophys Res Commun. 2006; 351: 185-91.

42. Sokolenko AP, Bulanova DR, Iyevleva AG, Aleksakhina SN, Preobrazhenskaya EV, Ivantsov AO, et al. High prevalence of GPRC5A germline mutations in BRCA1-mutant breast cancer patients. Int J Cancer. 2014; 134: 2352-8.

43. Wu Q, Ding W, Mirza A, Van Arsdale T, Wei I, Bishop WR, et al. Integrative genomics revealed RAI3 is a cell growth-promoting gene and a novel P53 transcriptional target. J Biol Chem. 2005; 280: 12935-43.

44. Liang M, Huang G, Liu Z, Wang Q, Yu Z, Liu Z, et al. Elevated levels of hsa_circ_006100 in gastric cancer promote cell growth and metastasis via miR-195/GPRC5A signalling. Cell Prolif. 2019; 52: e12661. 
45. Mi L, Lei L, Yin X, Li N, Shi J, Han X, et al. Circ_0000144 functions as a miR-623 sponge to enhance gastric cancer progression via up-regulating GPRC5A. Biosci Rep. 2020; 40: BSR20201313.

46. Sawada Y, Kikugawa T, Iio H, Sakakibara I, Yoshida S, Ikedo A, et al. GPRC5A facilitates cell proliferation through cell cycle regulation and correlates with bone metastasis in prostate cancer. Int J Cancer. 2020; 146: 1369-82.

47. Moyano-Galceran L, Pietila EA, Turunen SP, Corvigno S, Hjerpe E, Bulanova $\mathrm{D}$, et al. Adaptive RSK-EphA2-GPRC5A signaling switch triggers chemotherapy resistance in ovarian cancer. EMBO Mol Med. 2020; 12: e11177.

48. Chen Y, Deng J, Fujimoto J, Kadara H, Men T, Lotan D, et al. Gprc5a deletion enhances the transformed phenotype in normal and malignant lung epithelial cells by eliciting persistent Stat 3 signaling induced by autocrine leukemia inhibitory factor. Cancer Res. 2010; 70: 8917-26.

49. Zhong S, Yin H, Liao Y, Yao F, Li Q, Zhang J, et al. Lung Tumor Suppressor GPRC5A Binds EGFR and Restrains Its Effector Signaling. Cancer Res. 2015; 75: 1801-14.

50. Fujimoto J, Nunomura-Nakamura S, Liu Y, Lang W, McDowell T, Jakubek Y, et al. Development of Kras mutant lung adenocarcinoma in mice with knockout of the airway lineage-specific gene Gprc5a. Int J Cancer. 2017; 141: 1589-99.

51. Daouk R, Hassane M, Bahmad HF, Sinjab A, Fujimoto J, Abou-Kheir W, et al. Genome-Wide and Phenotypic Evaluation of Stem Cell Progenitors Derived From Gprc5a-Deficient Murine Lung Adenocarcinoma With Somatic Kras Mutations. Front Oncol. 2019; 9: 207.

52. Ma X, Schwarz A, Sevilla SZ, Levin A, Hultenby K, Wernerson A, et al. Depletion of Gprc5a Promotes Development of Diabetic Nephropathy. J Am Soc Nephrol. 2018; 29: 1679-89.

53. Wacker D, Stevens RC, Roth BL. How Ligands Illuminate GPCR Molecular Pharmacology. Cell. 2017; 170: 414-27.

54. Brauner-Osborne H, Krogsgaard-Larsen P. Sequence and expression pattern of a novel human orphan G-protein-coupled receptor, GPRC5B, a family C receptor with a short amino-terminal domain. Genomics. 2000; 65: 121-8.

55. Ceyhan GO, Demir IE, Altintas B, Rauch U, Thiel G, Muller MW, et al. Neural invasion in pancreatic cancer: a mutual tropism between neurons and cancer cells. Biochem Biophys Res Commun. 2008; 374: 442-7.

56. Hirai I, Kimura W, Ozawa K, Kudo S, Suto K, Kuzu H, et al. Perineural invasion in pancreatic cancer. Pancreas. 2002; 24: 15-25.

57. Pour PM, Bell RH, Batra SK. Neural invasion in the staging of pancreatic cancer. Pancreas. 2003; 26: 322-5.

58. Ceyhan GO, Bergmann F, Kadihasanoglu M, Altintas B, Demir IE, Hinz U, et al. Pancreatic neuropathy and neuropathic pain--a comprehensive pathomorphological study of 546 cases. Gastroenterology. 2009; 136: 177-86 e1.

59. Friess H, Shrikhande S, Shrikhande M, Martignoni M, Kulli C, Zimmermann $A$, et al. Neural alterations in surgical stage chronic pancreatitis are independent of the underlying aetiology. Gut. 2002; 50: 682-6.

60. Liebl F, Demir IE, Mayer K, Schuster T, D'Haese JG, Becker K, et al. The impact of neural invasion severity in gastrointestinal malignancies: a clinicopathological study. Ann Surg. 2014; 260: 900-7; discussion 7-8.

61. Kato S, Kubota K, Shimamura T, Shinohara Y, Kobayashi N, Watanabe S, et al. Semaphorin 4D, a lymphocyte semaphorin, enhances tumor cell motility through binding its receptor, plexinB1, in pancreatic cancer. Cancer Sci. 2011; 102: 2029-37.

62. Patnaik A, Weiss GJ, Leonard JE, Rasco DW, Sachdev JC, Fisher TL, et al. Safety, Pharmacokinetics, and Pharmacodynamics of a Humanized Anti-Semaphorin 4D Antibody, in a First-In-Human Study of Patients with Advanced Solid Tumors. Clin Cancer Res. 2016; 22: 827-36.

63. Zuazo-Gaztelu I, Paez-Ribes M, Carrasco P, Martin L, Soler A, Martinez-Lozano M, et al. Antitumor Effects of Anti-Semaphorin 4D Antibody Unravel a Novel Proinvasive Mechanism of Vascular-Targeting Agents. Cancer Res. 2019; 79: 5328-41.

64. Bulanova DR, Akimov YA, Rokka A, Laajala TD, Aittokallio T, Kouvonen P, et al. Orphan G protein-coupled receptor GPRC5A modulates integrin beta1-mediated epithelial cell adhesion. Cell Adh Migr. 2017; 11: 434-46.

65. Vodrazka P, Korostylev A, Hirschberg A, Swiercz JM, Worzfeld T, Deng S, et al. The semaphorin $4 \mathrm{D}$-plexin-B signalling complex regulates dendritic and axonal complexity in developing neurons via diverse pathways. Eur J Neurosci. 2009; 30: 1193-208.

66. Richter L, Oberlander V, Schmidt G. RhoA/C inhibits proliferation by inducing the synthesis of GPRC5A. Sci Rep. 2020; 10: 12532

67. Saitoh M, Endo K, Furuya S, Minami M, Fukasawa A, Imamura T, et al. STAT3 integrates cooperative Ras and TGF-beta signals that induce Snail expression. Oncogene. 2016; 35: 1049-57.

68. Komiya Y, Habas R. Wnt signal transduction pathways. Organogenesis. 2008; 4: 68-75.

69. Piccolo S, Dupont S, Cordenonsi M. The biology of YAP/TAZ: hippo signaling and beyond. Physiol Rev. 2014; 94: 1287-312. 